local guidelines. He attained his normal milestones with no delay, he crawled at one year and walked at 14 months, and he has 20 words with understanding of 3 steps command and respond appropriately. The delivery was full term vaginal delivery with no complications. On initial clinical examination he had no facial asymmetry or any other cranial nerve palsies. There was slight movement against gravity in the right upper limb compared to the left one and his right hand was in continuous fist. Tone in the right upper limb increased compared to the left upper limb with normal reflexes. His gait was normal with no tendency to fall to either side. Extensive laboratory investigations were done, full blood count initially showed leucocytosis with high eosinophilia. CT scan was done and it showed subtle low attenuation in the left globus pallidus. In addition, MRI under general anaesthesia demonstrated a small area of diffusion restriction in the left globs pallidus which indicated ischemia. MR angiography revealed narrowing of anterior cerebral artery and middle cerebral artery compared to the right side. Lumbar puncture was done and it was negative for varicella zoster PCR.

Discussion Cerebral complications post varicella infection is a cause of stroke in children. Detailed history of previous infection is essential in making a clinical diagnosis. One literature review states that $44 \%$ of cerebrovascular event in children is a consequence/associated with varicella cerebral complications.

\section{GP70 PULMONARY MILIARY TUBERCULOSIS IN A TODDLER AFTER INITIAL PRESENTATION WITH TUBERCULOUS MENINGITIS}

${ }^{1}$ Ancuta Bilasco, ${ }^{2}$ Ramona Cirt*, ${ }^{3}$ Szidonia Florea, ${ }^{1}$ Anca Draganescu, ${ }^{1}$ Magda Vasile, ${ }^{1}$ Camelia Kouris, ${ }^{1}$ Cristina Negulescu, ${ }^{4,1}$ Monica Luminos. ${ }^{1}$ National Institute for Infectious Diseases 'Prof. Dr. Matei Balş', Bucharest, Romania; ${ }^{2}$ Bucharest Oncology Institute 'Profesor Doctor Alexandru Trestioreanu', Bucharest, Romania; ${ }^{3}$ Children's Emergency Hospital, ClujNapoca, Romania; ${ }^{4}$ Carol Davila University of Medicine and Pharmacy, Bucharest, Romania

\subsection{6/archdischild-2019-epa.136}

Introduction Even though tuberculosis is considered to be a rare disease in developed countries, Romania is facing an important public health problem despite the fact that every newborn receives a BCG vaccine in maternity.

Case report We report a case of a 1 year and 9 months age female toddler from the roma community, otherwise previously healthy, born outside of Romania therefore she was not immunized against tuberculosis who presented with fever, choreiform movements, malaise, fatigue, anorexia, vomiting and generalized seizure. Clinical assessment showed positive signs of meningeal irritation and a Glasgow Coma Score of 8-9/15. CSF studies showed lymphocytosis and decreased glucose suggesting TB etiology. Thoracic radiography revealed the presence of a right hilar adenopathy and confluent right pericardial lesions. Head CT showed internal hydrocephalus and right maxillary sinusitis confirmed by MRI which also revealed areas of infarction and multiple inflammatory lesions suggestive of meningeal inflammation. M. tuberculosis was not detected in CSF PCR and cultures were negative. Considering close household contact with uncle and grandmother with active TB disease, the diagnosis of TB meningoencephalitis was made. Treatment with tetra conjugated tuberculostatics, corticosteroids and anticonvulsants was started. The patient continued to deteriorate so she had a temporary external CSF drainage placed. In evolution she developed cerebral venous and arterial thrombosis and she was started on anticoagulants. Consequently, she developed cortical blindness. Despite all this her state gradually improved and she was discharged after 70 days to follow anticonvulsant and tuberculostearic treatment at home but she was lost to follow up. After 9 months the mother interrupted treatment by her own will and she returned to the hospital a year after the first admission with high fever and presenting generalized seizures. Neurologic assessment showed a delay in language and mental retardation, normal sight. Thoracic radiography revealed hilar adenopathy and miliary aspect. Etiologic treatment was initiated and she was discharged to continue antituberculous treatment being closely monitored by a pneumologist, with favorable outcome.

Discussion The case has an emotional and social impact, the patient coming from the roma community, without social security number thus impossibility of receiving treatment on the national programme, unimmunized, raising the question of BCG vaccination of certain ethnic groups regardless of the country of birth.

\section{GP71 CASE SERIES: DIFFERENT AETIOLOGIES OF CHILDHOOD PERICARDITIS}

Fauzia Akhtar*. Rani Wasala. Wexford General Hospital, Wexford, Ireland

\subsection{6/archdischild-2019-epa.137}

Aim This is a study of case series to find out the underlying causes of pericarditis in children presenting to the paediatric ward of a District General Hospital in Ireland.

Methods We encountered 4 patients who were diagnosed to have pericarditis in the Department of Paediatrics of Wexford General Hospital between the months of March to July 2018. Their medical records were studied to look for signs and symptoms at initial presentation, investigations carried out in our hospital as well as at the tertiary centre, the management done and outcome.

Results The study of medical records of these four patients showed that one of the patients had Non Hodgkin Lymphoma as an underlying cause; in the second patient pericarditis was the presenting feature of systemic onset juvenile rheumatoid arthritis. Rest of the two patients' cause was unknown and were classified as idiopathic.

All four patients had their ECG and echocardiogram done which confirmed the diagnosis of pericarditis. The patients were referred to the tertiary cardiac unit for further management. 2 cases with idiopathic pericarditis did improve with few weeks of treatment with ibuprofen and the other two cases received treatment for the underlying conditions which resulted in the improvement of the symptoms and sings related to pericarditis and the underlying illnesses.

Discussion This showed that the most common cause of pericarditis in paediatric age group is idiopathic which is in accordance with the published literature. The other causes found were secondary to malignancy and rheumatological condition. The symptoms and signs of pericarditis did improve with ibuprofen and commencement of treatment of the underlying causes.

Conclusion The most common cause of pericarditis in paediatric population is idiopathic. The outcome is dependent upon the underlying causes and is generally favourable. 